\title{
Study of a Small Amoeba from Mammalian Cell Cultures Infected with 'Ryan Virus'
}

\author{
By D. C. WARHURST AND J. A. ARMSTRONG \\ National Institute for Medical Research, Mill Hill, London, N.W. 7
}

(Accepted for publication I 2 July 1967)

\begin{abstract}
SUMMARY
A small amoeboid organism, found in mammalian tissue cultures inoculated with an infective agent formerly termed 'Ryan virus', is shown to have the morphological, cultural and behavioural characters of the freeliving soil amoeba Hartmannella castellanii Douglas, 1930. Cytopathic changes occurred regularly in the infected monolayers; this was evidently due to action of the amoebae rather than the presence of any associated bacterial or viral agents. Strong circumstantial evidence suggests that the Ryan isolates of $H$. castellanii originated, either as trophozoites or cysts, from swabs of the human nasopharynx. Recovery of hartmannellid amoebae from this source is of interest in relation to some recently reported cases of pyogenic meningitis, apparently caused by free-living soil amoebae.
\end{abstract}

\section{INTRODUCTION}

A transmissible cytopathic agent, provisonally called 'Ryan virus', was isolated in several laboratories by inoculation of tissue cultures with swabs of the upper respiratory tract from patients showing fever and respiratory symptoms (Pereira, Marsden Corbitt \& Tobin, 1966). Some properties of the agent seemed to be virus-like, including cytopathic changes with intranuclear inclusions observed in all inoculated cultures. Subsequent cytological studies by light- and electron-microscopy indicated that the cytopathic effects were attributable, not to a virus, but to a motile cellular organism resembling the free-living soil amoeba Hartmannella castellanii (Armstrong \& Pereira, 1967). In the present paper further details are given of the evidence leading to identification of the organism, including cultivation in the absence of mammalian cells, and morphological and behavioural characteristics distinguishing hartmannellid amoebae from somewhat similar free-living forms of the family Schizopyrenidae.

\section{MATERIALS AND METHODS}

The agent described in this paper was the original isolate of the series reported by Pereira et al. (I966) and designated 'Ryan I'. HeLa cell cultures infected with Ryan I were kindly supplied by Dr M.S. Pereira (Virus Reference Laboratory, Central Public Health Laboratory, Colindale). HeLa cell monolayers were grown at $37^{\circ}$ in stationary tubes containing coverslips, using a medium composed of Gey balanced salt solution containing $10 \%(\mathrm{v} / \mathrm{v})$ human serum and $0.5 \%(\mathrm{w} / \mathrm{v})$ lactalbumin hydrolysate with 100 units of penicillin and $100 \mu \mathrm{g}$. streptomycin $/ \mathrm{ml}$. For microscopy, monolayers were fixed in Bouin fluid, and stained with haematoxylin and eosin.

Two procedures were adopted for selective cultivation of amoebae. (I) Petri-dish 
plates prepared from $1 \cdot 5 \%(w / v)$ Ionagar (Oxoid: Oxo Ltd., Thames House, Queen St Place, London, E.C. 4) were spread with a suspension in saline of Klebsiella aerogenes (NCTC 9667) which had been grown in broth and killed by heating at $56^{\circ}$ for $2 \mathrm{hr}$. Surface water was dried from the plates, they were spread with drops of the supernatant fluid of cell cultures infected with Ryan $I$, and were then incubated at $36-37^{\circ}$. (2) For growth of pure (axenic) cultures, supernatant fluid of cell cultures infected with Ryan I, or amoebae grown on agar plates as shown, were inoculated into either Neff (1957) proteose-peptone glucose medium in $5 \mathrm{ml}$. quantities in sloped McCartney bottles or into a modified $4 \%(\mathrm{w} / \mathrm{v})$ mycological peptone (Adam, 1964) and incubated at $36.5^{\circ}$. The latter medium was prepared as follows: mycological peptone (Oxoid) was dissolved in $\mathrm{M} / \mathrm{I} 5$ phosphate buffer $(\mathrm{pH} 7)$. The solution was boiled to allow a precipitate to form, and then filtered. The filtrate was autoclaved in $5 \mathrm{ml}$. quantities in McCartney bottles for $20 \mathrm{~min}$. at $\mathrm{I} 2 \mathrm{I}^{\circ}$. The bottles were sloped after inoculation, and subcultures were made at intervals of 4-7 days.

For studies of encystment and mitosis, permanent preparations were made by growing amoebae with living or killed Klebsiella aerogenes on microscope slides, coated with a thin layer of agar, in a moist atmosphere; the cultures were fixed in situ using Bouin or Carnoy fixative (Singh, 1950). Singh's technique was slightly modified by cutting small slits in the agar layer during inoculation of amoebae on to the slide. This enabled the amoebae to reach the glass surface more readily and to migrate under the agar. The agar layer was stripped off after fixation, and the amoebae adhering to the slide were washed in $70 \%(\mathrm{w} / \mathrm{w})$ ethanol in water followed by distilled water, mordanted for $18 \mathrm{hr}$ in $3 \%(\mathrm{w} / \mathrm{v})$ ferric alum, washed in distilled water, and stained for $4-18 \mathrm{hr}$ in $0.5 \%(\mathrm{w} / \mathrm{v})$ haematoxylin. The stain was differentiated with $\mathrm{I} \%(\mathrm{w} / \mathrm{v})$ ferric alum, and the preparations were counterstained, before final dehydration, in $2 \%(w / v)$ ethanolic aqueous eosin solution. Other preparations were made by allowing a drop of culture fluid containing many amoebae to lie on a slide for about $30 \mathrm{~min}$. in a moist atmosphere, and then running on fixative to fix the amoebae which adhered to the slide. The Feulgen test was done according to Singh (1952).

In tests to determine whether the amoebae would transform into flagellates, the surface of an agar-plate culture of amoebae was flooded with distilled water, and observations were made at intervals of $\mathrm{I}, 2,4,6,9$ and $24 \mathrm{hr}$ by using an inverted microscope. In another experiment, axenic cultures were centrifuged and the $5 \mathrm{ml}$. of supernatant fluid discarded. The deposited amoebae were suspended in Io $\mathrm{ml}$. de-ionized water and samples were pipetted into Petri dishes for observation with an inverted microscope.

\section{RESULTS}

On observation with the inverted microscope of living HeLa cell monolayers, infected with Ryan I, small amoebae were seen to move slowly across the cell sheet, sometimes remaining for a considerable time in one position close to a particular HeLa cell. Focal damage to the cell sheet was obvious to the naked eye 2 days after inoculation, and complete stripping of the monolayer often occurred by 4 days. Cytological features of the cytopathic effect due to Ryan isolates in tissue cultures were described by Armstrong \& Pereira (1967), with particular reference to early changes involving the nuclei and nucleoli of the affected cells. Encystment of the amoebae with consequent detachment from the glass surface also occurred regularly, and cysts 
were readily detected by microscopical inspection of the supernatant culture medium after 2-3 days of infection.

In agar-plate cultures, single amoebae could be detected after a few hours by using the inverted microscope. They left behind them tortuous trails in moving through the accumulated bacteria on the agar surface. At the end of each trial there could usually be found an indistinct amoeba with a clearly visible contractile vacuole emptying at intervals of about $2 \mathrm{~min}$. When the agar surface had become drier, colonies of amoebae developed which were detectable by the naked eye as clear plaques on the confluent bacterial surface layer, similar to the plaques described for Hartmannella castellanii by Van Rooyen (1932 $a, b)$. Within about 4 days, or earlier in a dry atmosphere, most of the amoebae had encysted.

In $4 \%$ mycological peptone a heavy growth of amoebae (about $6 \times 10^{4} / \mathrm{ml}$.) was produced after 4-7 days, which could be detected by viewing the culture with the naked eye in subdued daylight, when finely granular clouds of amoebae could be seen in the fluid as it was gently shaken. Examination of cultures by using the inverted microscope disclosed amoebae floating in the medium and adhering to the glass. Cysts were produced in this system after about 6 days, but the agar-plate method produced the maximum yields of cysts. The axenic cultures were subcultured by adding $0 \cdot I-$ $0.5 \mathrm{ml}$. of a well-mixed 4 - to 7 -day-old culture to each fresh culture bottle. The inoculum could be stored for more than a month at $4^{\circ}$ without appreciable loss of viability.

\section{Morphology of the trophozoites}

The cytoplasm consisted of an inner granular endoplasm, containing the cell organelles, and an outer hyaline ectoplasm. The pseudopodia were fine spike-like, or large lobose, extensions of the ectoplasm, which were often formed simultaneously. Movement of the organism was slow, but appeared to be slightly more rapid in the presence of tissue culture cells. Fifty living amoebae from an axenic culture in $4 \%$ mycological peptone at $36.5^{\circ}$ were measured by using an eyepiece micrometer, and their mean diameter when rounded was I $8 \cdot 8 \mu$ (range: $12 \cdot 3-27 \cdot 7 \mu)$. In fixed and stained preparations some shrinkage occurred, and the mean diameter was $14 \mu$. Amoebae grown on agar surfaces with Klebsiella aerogenes (Pl. I, figs. I, 2) were larger than amoebae from HeLa cell cultures or from pure cultures. There was a single contractile vacuole, situated at the rear end during movement of the organism. Although amoebae were occasionally found which had 2 or more nuclei, there was generally only one nucleus, which was spherical and vesicular, with a delicate nuclear membrane. In life it was often $7 \mu$ or more in diameter. The mean diameter in fixed and stained cultures with HeLa cells was $3.5 \mu$ (range of 62 nuclei: $2 \cdot 7-5 \cdot 4 \mu$ ). Amoebae grown on agar surfaces with $K$. aerogenes had a mean nuclear diameter, when fixed and stained, of $5 \cdot 3 \mu$ (range of 50 nuclei: $3 \cdot 4-6 \cdot 8 \mu$ ). There was a strongly basophilic spherical karyosome or nucleolus, which often appeared hollow; it gave a negative Feulgen reaction. The nucleolar diameter was usually just over half that of the nucleus. The mean diameter of nucleoli from fixed and stained cultures with HeLa cells was $2 \cdot 0 \mu$ (range of 44 nucleoli: $1 \cdot 4-2 \cdot 7 \mu$ ). In fixed and stained amoebae from cultures on agar surfaces, the mean nucleolar diameter was $3 \cdot 2 \mu$ (range of 50 nucleoli : $2 \cdot 0-4 \cdot 1 \mu$ ). Slightly basophilic granules, just inside the nuclear membrane, gave a positive Feulgen staining reaction. These granules evidently represent the desoxyribonucleoprotein component of the nucleus. 
Cyst formation and morphology. When the cysts were first formed they were rounded, with a thin wall, which became thicker and wrinkled when they ripened. The wall was then double-contoured and pierced by several circular ostioles (as many as 8), each sealed with an operculum. The ostioles were most easily visible in empty cysts (Pl. 2, figs. $7,8,9$ ). The mean diameter of 50 living cysts from a pure culture was $18.6 \mu$ (range $\mathrm{I} 2 \cdot 3-30 \cdot 8 \mu$ ). There was usually a single nucleus similar to that of the trophozoite. The peripheral part of the cytoplasm in the living cysts was occupied by a layer of dense granules. In excystment, which occurred in the presence of bacteria or HeLa cells, the amoeba apparently emerged through one of the ostioles, after dislodging the operculum (P1. 2, figs. 8, 9). On agar plates smeared with Klebsiella aerogenes, most cysts had hatched in less than $6 \mathrm{hr}$ at $36.5^{\circ}$. Cysts which had been kept under dry conditions at $20^{\circ}$ for more than 3 months hatched readily when inoculated on to agar plates spread with $K$ aerogenes. Using viability as a test of survival, it was found that cysts were killed by exposure (for $30 \mathrm{~min}$. at $20^{\circ}$ ) to methanol, $5 \%(\mathrm{v} / \mathrm{v})$ Lysol, $70 \%$ $(\mathrm{v} / \mathrm{v})$ ethanol or $10 \%(\mathrm{v} / \mathrm{v})$ formol saline. Under the same conditions, neither distilled water nor $0.3 \%(\mathrm{w} / \mathrm{v})$ Pyroneg (a commercial glassware cleaner obtained from Diversey (U.K.) Ltd., 42-46 Weymouth Street, London, W. I) killed the cysts.

Mitotic division. During mitosis the amoebae became round and ceased to move. In prophase the peripheral Feulgen-positive granules of the nucleus condensed to form chromosomes, whilst the nucleolus began to decrease in size (P1. I, fig. 3). When the nucleolus had completely disappeared, spindle fibres appeared, originating apparently from areas of the nuclear membrane which had broken down (Pl. I, fig. 4). No centrioles were visible at any stage. The nuclear membrane then broke down, whilst the chromosomes became arranged on the equatorial plate and a typical metaphase figure developed (Pl. I, fig. 5). When the daughter chromosomes moved apart in anaphase, they became more basophilic (P1. I, fig. 6). New nuclear membranes formed and two interphase nuclei were reconstituted before cytoplasmic division took place.

Flagellate stage. No flagellate stages were found when amoebae were suspended in distilled or de-ionized water for various lengths of time, although some amoebae disintegrated, some encysted, and some continued to move about for more than 2 days.

Cytopathogenicity of amoebae grown with bacteria and in pure cultures. Amoebae which had been subcultured 4 times on agar surfaces, with dead Klebsiella aerogenes as food, were suspended in saline and 3 serial Io-fold dilutions $\left(\mathrm{IO}^{-1}, \mathrm{IO}^{-2}, \mathrm{IO}^{-3}\right)$ were inoculated into cultures of HeLa cells in stationary tubes with coverslips. Cytopathic effects beginning with nuclear changes typical of those reported for the Ryan isolates developed in all the inoculated cultures and led to destruction of the cell sheet. Parallel uninoculated cultures showed no such effects.

Amoebae grown axenically in Neff medium were similarly effective in producing the typical cytopathic effect in cultures of HeLa cells. These results, together with the absence of recognizable virus particles or of other regularly associated microbial agents in sections of the amoebae or infected tissue cultures examined by electron microscopy (Armstrong \& Pereira, 1967) suggest that the hartmannellid amoebae were themselves responsible for the cytopathic effect produced by Ryan isolates in cultured mammalian cells. Moreover, the Ryan agent would not pass through filters which admitted viral agents. Further work on the nature and mechanism of the cytopathic effect is in progress. 


\section{DISCUSSION}

\section{Taxonomy}

Amoebae of the 'Naegleria' or 'Vahlkampfia' type (family Schizopyrenidae, Singh, I952) produce the elongated 'Limax' form readily, generally move rapidly in fresh cultures, and may produce flagellate forms when suspended in distilled water. Their mitosis in the trophozoite stage is distinctive, for the nucleolus is retained and divides to form 'polar masses' (Fig. I A). Hartmannellid amoebae (family Hartmannellidae, Singh, 1952) generally move more slowly, and filiform or spike-like pseudopodia are often present. They do not produce flagellate forms. Their mitotic cycle is similar to that found in higher animals and plants. The nucleolus is lost, and a spindle develops, with chromosomes arranged on the equatorial plate (Fig. I B). The mitotic cycle, the absence of a flagellate stage, the pseudopodia, the body form and the slow locomotion of the Ryan I amoeba are all characteristic of the family Hartmannellidae.
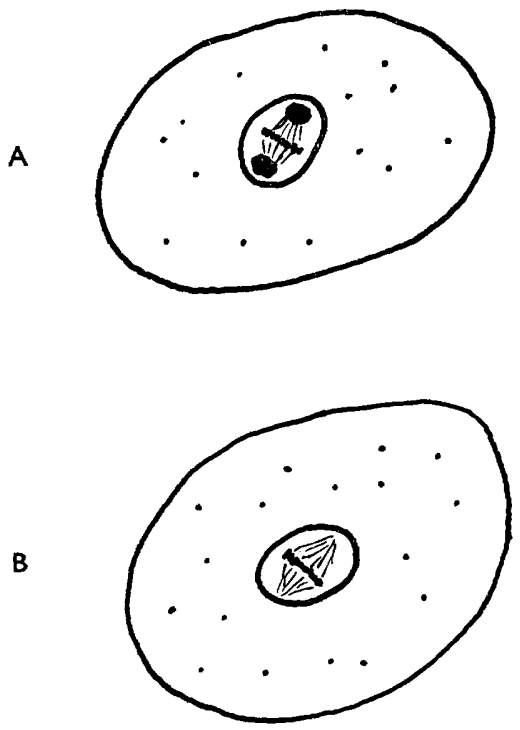

Fig. I. Diagrammatic comparison of the metaphase figure of mitosis (as if stained with haematoxylin) in (A) a schizopyrenid amoeba and (B) a hartmannellid amoeba.

The structure of the nucleus, with a single large Feulgen-negative karyosome (nucleolus) and peripheral Feulgen-positive material, the absence of nuclear division in the cysts, the aerobic mode of growth and the size-range of the trophozoites separate the Ryan I amoeba from the genera in Hartmannellidae other than the genus Hartmannella Alexeieff 1912 $a, b$, as described by Singh (1952) and Adam (1964). [Singh (1952) and Adam (1964) considered that Volkonsky (I93I) split off 'Acanthamoeba' from the genus Hartmannella on inadequate criteria; with this we concur.] The Ryan I amoeba accords well with the descriptions of $H$. castellanii Douglas (1930) given by Castellani (I930 $a-d$ ), Douglas (1930), Volkonsky (I93I) and Adam (I964), apart from our inability to find a centriole as described by Volkonsky. Three of the four species of free-living hartmannellid amoeba described by Singh (1952) have a different cyst morphology from the Ryan I amoeba; $H$. rhysodes Singh, I952, is closely similar 
to Ryan I, but we agree with the opinion of Adam (1964) that the name $H$. rhysodes is a synonym of $H$. castellanii. There is little doubt therefore that the Ryan I amoeba is a strain of $H$. castellanii. In the table we give a comparison of the diagnostic features of Ryan I and $H$. castellannii at the species level.

Table I. Comparison of the Ryan I amoebae with Hartmannella castellanii Douglas 1930

Body form

Diameter in life, 7.2-37.0 $\mu$ (mean I $8 \cdot 1 \mu$ ). Very slow progression, 'Limax' form rare. Spikelike and lobose pseudopodia. Single contractile vacuole (period 56-I $37 \mathrm{sec}$.

Diameter in life

12.3-27.7 $\mu$ (mean $18 \cdot 8 \mu)$. Very slow progression. 'Limax' form not found. Spike-like and lobose pseudopodia. Single contractile vacuole (period $\mathrm{I} 20 \mathrm{sec}$.)

* Castellani (1930a-d), Douglas (1930), Volkonsky (193I), Adam (1964).
On bacteria and yeasts, dead or alive, from $25^{\circ}$ to $35^{\circ}$. Growth in peptone and defined media below $35^{\circ}$

On bacteria, dead or alive, at up to $37^{\circ}$. With tissue culture cells up to $37^{\circ}$. Growth in peptone media at $36.5^{\circ}$

Our inability to demonstrate an intranuclear centriole in Ryan I amoebae may well be unimportant since the reported size of this organelle is at the limit of resolution of the light microscope, and its very existence in small amoebae is already disputed (Singh, 1952). With regard to the temperature requirements of different strains, Adam (I964) found that although the Castellani strain of Hartmannella castellanii would not grow in $4 \%$ mycological peptone at $35^{\circ}$, is of the 20 similar strains of hartmannellid amoebae she studied grew well at this temperature. She pointed out that many strains grow readily at (or near) mammalian body temperature.

The various observations reported in our paper refer directly to only one of the tissue culture isolates, namely Ryan I, of the series described by Pereira et al. (1966). Nevertheless, it has been confirmed that all isolates of Ryan agent contained similar free-living amoebae (Armstrong \& Pereira, 1967), and the cytopathic picture in each case was identical. It is therefore highly probable that all of the Ryan isolates were strains of Hartmannella castellanii. Hartmannellid amoebae previously recovered by Wang \& Feldman (196I) from cell cultures inoculated with material from human pharyngeal swabs, were considered by these authors to include $H$. rhysodes, $H$. glebae and $H$. agricola. They described the cyst of the form they considered to be $H$. rhysodes, and it is clearly comparable to that of $H$. castellanii and the Ryan I amoeba. The recent work of Chang, Hung Pan \& Rosenau (1966) is relevant to the discussion of cytopathogenicity of small amoebae and their presence in tissue cultures. These authors consider the possibility that an infective agent known for several years as 'lipovirus', which was recovered in liver-cell cultures inoculated with human hepatitis 
serum (Chang, I96I), is associated with a small amoeboid cell. Certainly the known properties of 'lipovirus' and 'Ryan virus' are strikingly similar, and it is even possible that they are one and the same agent.

\section{Significance of Hartmannella castellanii in tissue cultures}

Free-living hartmannellid amoebae have been positively identified in mammalian tissue cultures on several occasions, although their precise origin was often obscure (Jahnes, Fullmer \& Li, 1957; Culbertson, Smith \& Minner, 1958; Adam, 1964). When trophozoites were detected in supposedly normal monkey kidney cell cultures, it seemed reasonable to regard them as contaminants derived from infected renal tissue or from airborne cysts in the laboratory. Wang \& Feldman (r96I), using HeLa and Hep-2 cell cultures, recovered amoebae from i9 out of I0,000 swabs of the human pharynx, the positive subjects being mostly children. Here, as well as in the more recent Ryan isolates in HeLa cell cultures, inherent or accidental contamination seemed an unlikely explanation; in the Ryan series, for example, amoebae consistently failed to appear in the parallel uninoculated control cultures. There is strong circumstantial evidence that these amoebae were of human origin. The patients concerned were suffering from fever and influenza-like symptoms of varying severity; however, it has yet to be shown with what frequency Hartmannella castellanii is recoverable from the throats of symptomless people in different age-groups, and there is little reason at present for supposing the amoebae to be concerned directly in human respiratory disease.

An important and as yet unanswered question in relation to the pathogenic potentialities of Hartmannella castellanii is whether the organisms are capable of persisting in the human nasopharynx in the trophic form, or occur merely as occasional cysts either inhaled by chance from the atmosphere or taken in by mouth. Eldridge \& Tobin (1967) by using a complement fixation test have found antibody (ranging in titre from $I$ in 5 to $I$ in 80) against Ryan isolates of Hartmannella in sera from 26 of I 28 people in hospital. The sera from a small group from whose throats hartmannellid amoebae had previously been isolated all fixed complement with Hartmannella antigen, although the titres fell to low values during a period of $\mathrm{I}-3$ years. These findings may well be indicative of an infection with trophic forms of the amoebae and also suggest that it is by no means a rare occurrence. Culbertson and his co-workers (1958, 1959, I96I) have shown conclusively that at least some strains of Hartmannella are pathogenic for mice and for monkeys, following inoculation of trophozoites by the intracerebral or intranasal routes; their experimental studies provide factual support for speculations concerning the possible role of free-living soil amoebae in human disease. Recent reports possibly incriminating small free-living amoebae in more serious disease include the demonstration of abundant trophozoites in post mortem brain sections from 4 rapidly fatal cases of pyogenic meningitis in Australia (Fowler \& Carter, 1965); furthermore, in 2 out of 3 similar cases in the United States (Butt 1966) the trophozoites were recognized, before death, in the cerebrospinal fluid.

We thank Dr Janet Niven, Dr F. Hawking and Dr C. G. Culbertson for encouragement and discussion. Drs M. S. and H. Pereira kindly supplied infected and uninfected HeLa cell cultures and also confirmed that amoebae grown axenically or with dead bacteria would produce the typical cytopathic effect. Mr J. B. Clark took the photomicrographs. 


\section{REFERENCES}

ADAM, K. M. G. (I964). A comparative study of Hartmannellid amoebae. J. Protozool. II, 423.

AleXEIEFF, A. (I912a). Sur les caractères cytologiques et la systématique des Amibes du groupe Limax. Bull. Soc. zool. Fr. 37, 55.

AleXeIEFF, A. (1912b). Quelques remarques supplémentaires sur la systématique des Amibes du groupe Limax. Sur le genre Sappina Dangeard. Bull. Soc. zool. Fr. 37, I49.

Armstrong, J. A. \& Pereira, M. S. (I967). Indentification of 'Ryan Virus' as an amoeba of the genus Hartmannella. Br. med. J. i, $2 \mathrm{I} 2$.

ButT, C. G. (1966). Primary amebic meningoencephalitis. New Engl. J. Med. 274, 1473.

Castellani, A. (1930a). An amoeba found in cultures of a yeast. Preliminary note. J. trop. Med. Hyg. $33,160$.

Castellani, A. (1930 b). An amoeba growing in cultures of a yeast. Second note. J. trop. Med. Hyg. 33, 188.

Castellani, A. (1930 c). An amoeba found in cultures of a yeast. Third note. J. trop. Med. Hyg. 33, 221.

Castellant, A. (1930d). An amoeba growing in cultures of a yeast. Fourth note. J. trop. Med. Hyg. 33, 237.

Chang, R. S. (196I). Properties of a transmissible agent capable of inducing marked DNA degradation and thymine catabolism in a human cell. Proc. Soc. exp. Biol. Med. I07, 135.

Chang, R. S., Hung Pan, I. \& Rosenau, B. J. (I966). On the nature of the 'Lipovirus' J. exp. Med. I24, I I 53 .

Culbertson, C. G. (I96I). Pathogenic Acanthamoeba. Am. J. clin. Path. 35, 195.

Culbertson, C. G., Smith, J. W. \& Minner, J. R. (1958). Acanthamoeba; observations on animal pathogenicity. Science, N.Y. 127, 1506.

Culbertson, C. G., Smith, J. W., Cohen, H. K. \& Minner, J. R. (1959). Experimental infection of mice and monkeys by Acanthamoeba. Am. J. Path. 35, I 85.

Douglas, M. (1930). Notes on the classification of the amoeba found by Castellani in cultures of a yeast-like fungus. J. trop. Med. Hyg. 33, 258.

ELdRIDGe, A. E. \& ToBin, J. O'H. (1967). 'Ryan Virus'. Br. med. J. i, 299.

Fowler, M. \& CARTER, R. F. (1965). Acute pyogenic meningitis probably due to Acanthamoeba sp. A preliminary report. Br. med.J. ii, 740 .

JAHNes, W. G., Fullmer, H. M. \& Li, C. P. (I957). Free living amoebae as contaminants in monkey kidney tissue culture. Proc. Soc. exp. Biol. Med. 96, 484.

NefF, R. J. (1957). Purification, axenic cultivation, and description of a soil amoeba, Acanthamoeba sp. J. Protozool. 4, 176.

Pereira, M. S., Marsden, H. B., Corbitt, G. \& Tobin, J., O’H. (I966). Ryan Virus; a possible new human pathogen. Br. med.J. i, I30.

SINGH, B. N. (I950). A culture method for growing small free-living amoebae for the study of their nuclear division. Nature, Lond. $\mathbf{6} 65,65$.

SINGH, B. N. (1952). Nuclear division in nine species of small free-living amoebae and its bearing on the classification of the order Amoebida. Phil. Trans. R. Soc. B 236, 405.

VAN ROOYEN, C. E. (1932a). The effect of Amoeba (Hartmannella) castellanii on bacterial cultures. J. trop. Med. Hyg. 35, 118.

VAN RoOYen, C. E. (I932 b). Observations on the clearing effect of Amoeba (Hartmannella) castellanii on bacterial cultures: a phenomenon simulating bacteriophagy. J. trop. Med. Hyg. 35, 259.

Volkonsky, M. (193I). Hartmannella castellanii Douglas et classification des Hartmannelles. Archs Zool. exp. gen. 72, 317.

WANG, S. S. \& Feldman, H. A. (196I). Occurrence of Acanthamoeba in tissue cultures inoculated with human pharyngeal swabs. In Antimicrobial Agents and Chemotherapy. vol. I, p. 50. Ed. by M. Finland and G. M. Savage. Michigan: Braun-Brumfield Inc. 

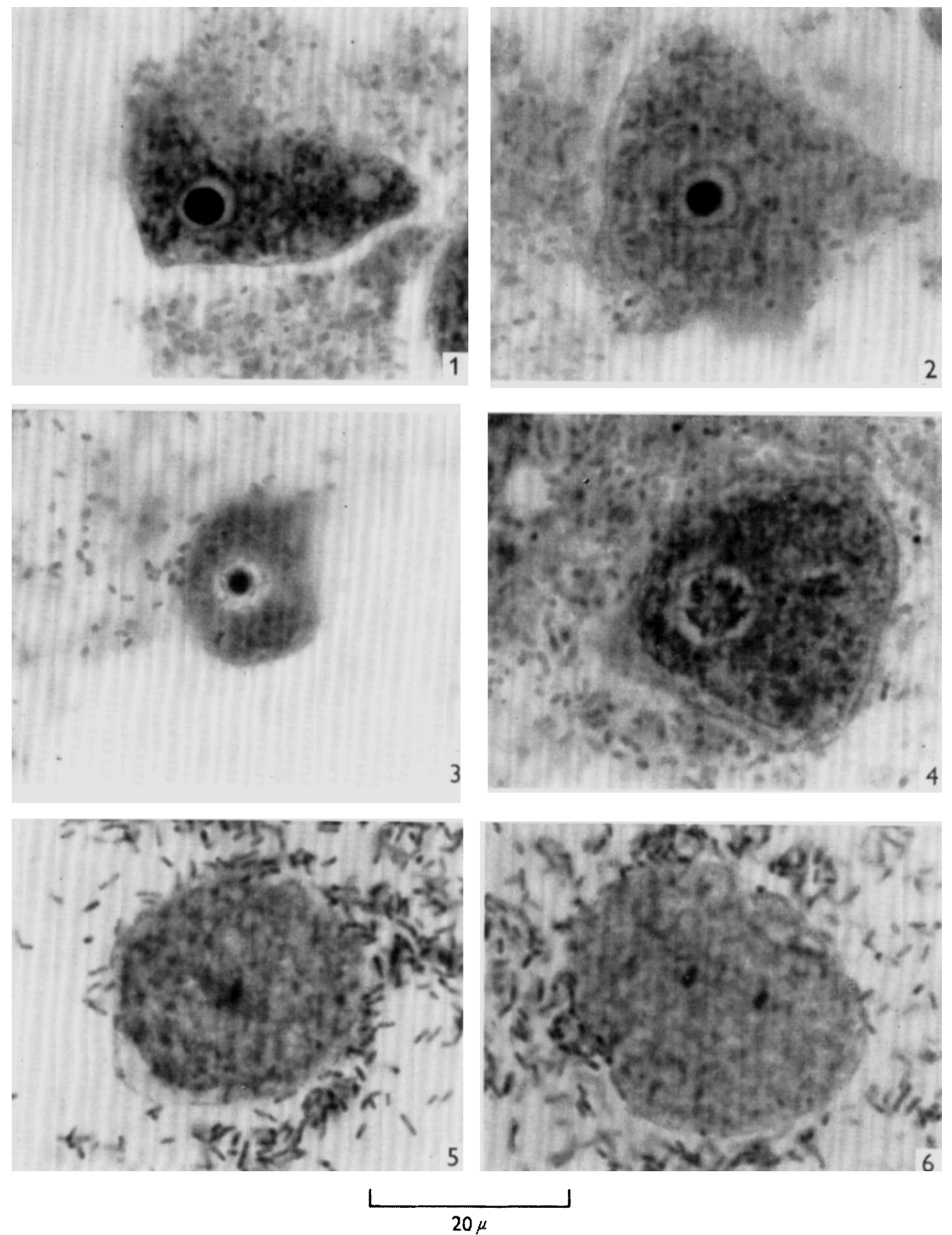

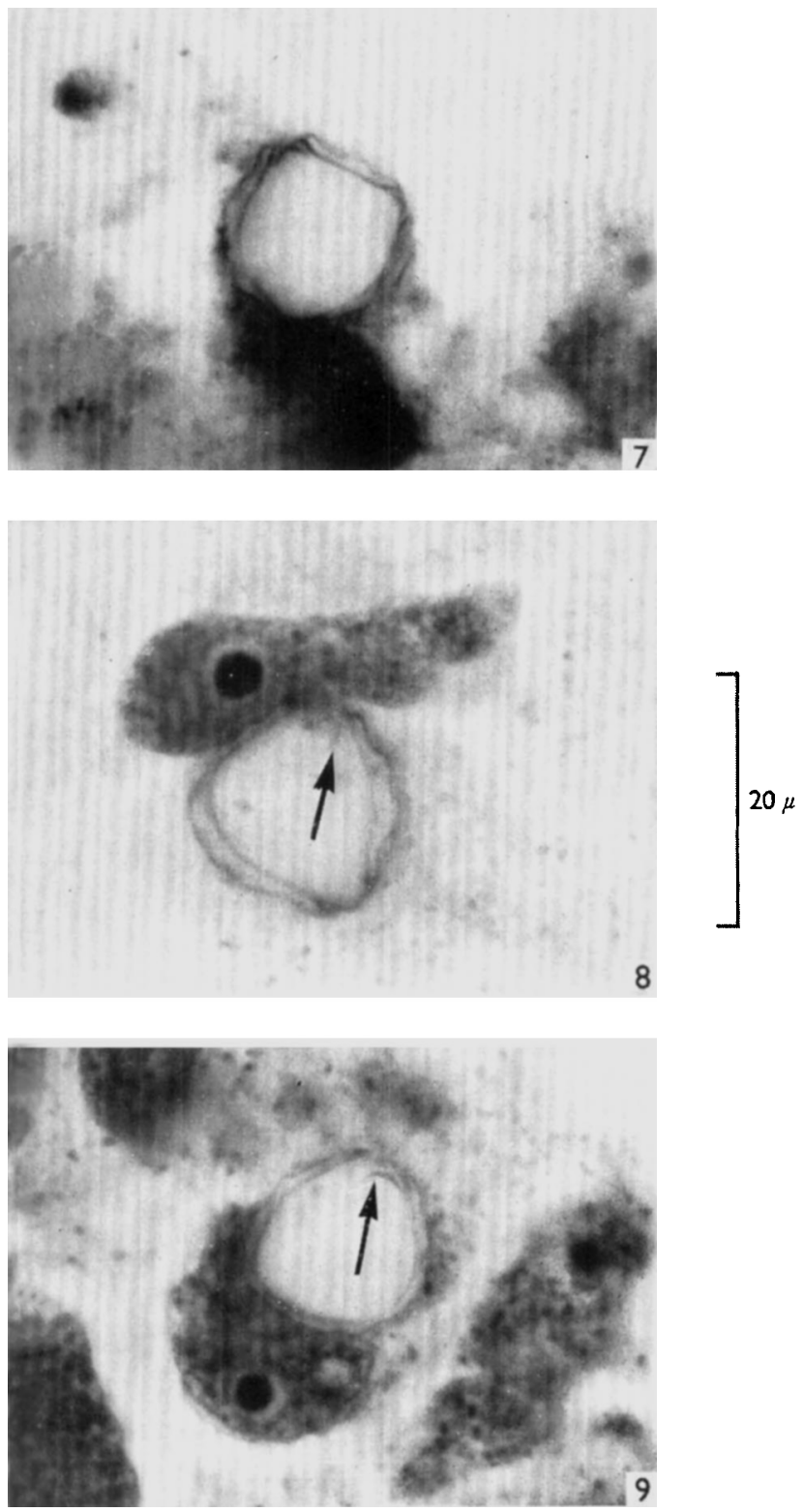

D. C. WARHURST AND J. A. ARMSTRONG 


\section{EXPLANATION OF PLATES}

Photomicrographs of permanent preparations of the Ryan I amoeba, grown with Klebsiella aerogenes on agar-coated slides. The organisms were fixed with Bouin's fluid and stained in iron alum haematoxylin, with ethanolic eosin as a counterstain. The scale represents $20 \mu$.

\section{Plate I}

Morphology and mitosis of the trophozoites

Fig. I. Interphase, showing the vesicular nucleus, with a densely staining single nucleolus, and the contractile vacuole.

Fig. 2. Interphase, showing the basophilic granules inside the nuclear membrane.

Fig. 3. Early prophase: the peripheral granules have condensed to form chromosomes, and the nucleolus is reduced in size.

Fig. 4. Late prophase: the nucleolus has disappeared whilst the spindle fibres and chromosomes are visible.

Fig. 5. Metaphase: chromosomes are arranged on the equatorial plate of the spindle. Note the absence of 'polar masses'.

Fig. 6. Anaphase: two groups of daughter chromosomes are moving apart. The spindle fibres are faintly visible.

Plate 2

Cyst-wall morphology in empty cysts

Fig. 7. Cyst showing the double-contoured wrinkled wall and 2 opercula in situ.

Fig. 8. Cyst showing dislodged operculum (arrow) and recently emerged (?) trophozoite.

Fig. 9. Cyst showing dislodged operculum (arrow) and recently emerged (?) trophozoite. 
